\title{
Gestión de mantenimiento en las gerencias de perforación y rehabilitación de la industria petrolera
}

\author{
Maintenance management in the drilling and rehabilitation management
} of the oil industry

\section{Jimmy John Ladino Soto \\ ladinoj88@gmail.com \\ https://orcid.org/0000-0002-5900-3375}

Petróleos de Venezuela S.A, Maracaibo - Venezuela
RESUMEN

Palabras clave
El propósito de la investigación fue analizar la gestión de mantenimiento en las gerencias de perforación y rehabilitación de la industria petrolera. Metodológicamente tuvo alcance descriptivo, con diseño no experimental, de campo y transeccional. La población quedó conformada por las 4 gerencias de perforación y rehabilitación de la industria petrolera de Venezuela, región occidente. Se empleó la encuesta como técnica mediante la aplicación de un cuestionario estructurado por 36 ítems con escala dicotómica. Para alcanzar la validez de este fue sometido al juicio de 5 expertos y se utilizó el coeficiente estadístico Küder Richardson para el cálculo de su confiabilidad, donde se obtuvo como valor de confiabilidad total del instrumento 0,94. Se concluye que estas gerencias ostentan buena gestión de mantenimiento. Situación que es producto del alto cumplimiento de las etapas del proceso y las estrategias de mantenimiento.

Gestión de mantenimiento; Industria petrolera; Planeación; Organización; Control
ABSTRACT

Keywords
The purpose of the research was to analyze maintenance management in the drilling and rehabilitation departments of the oil industry. Methodologically, it had a descriptive scope, with a non-experimental, field and transectional design. The population was made up of the 4 drilling and rehabilitation departments of the oil industry, western region. The survey was used as a technique by applying a questionnaire structured by 36 items with a dichotomous scale. To achieve the validity of this, it was submitted to the judgment of 5 experts and the statistical coefficient Küder - Richardson was used to calculate its reliability, where 0.94 was obtained as the total reliability value of the instrument. It is concluded that these managements have good maintenance management. Situation that is the product of high compliance with the process stages and maintenance strategies.

Maintenance management; Oil industry; Planning; Organization; Control 
INTRODUCCIÓN

Las fuerzas de mercado actuales exigen excelencia en los productos, por ello sería inconcebible que el mantenimiento, siendo función importante de apoyo a producción, y por ende parte de la organización empresarial, fuera deficiente. En este sentido, las empresas tienen el reto de mejorar sus actividades de gestión del mantenimiento para ser sostenibles. En este contexto, para Cáceres (2009), el mantenimiento como estructura de apoyo, es un centro de costos a efectos de los intereses de la empresa. Ciertamente, afirma este autor, como un costo, sólo se justifica si perfecciona el negocio a través de la mejora de las condiciones de productividad, mediante la capacidad continua de adaptación, desarrollo y conservación. Para ello, se debe enfocar adecuadamente la visión y misión mediante la definición clara de políticas, objetivos y valores, entre otros.

Ahora bien, sobre la base de lo referido por Duffuaa et al. (2010), es un hecho que, en los escenarios de hoy, las empresas se juegan su capacidad competitiva por la cantidad y calidad de los recursos que se comprometen en el área de mantenimiento, debido a la capacidad de estas para generar beneficios a su más inmediato grupo de interés como es, el área de producción. La principal ventaja que ofrece el mantenimiento reside en la consecución de que los sistemas productivos continúen desempeñando las funciones deseadas, y así contribuir a conservar las actividades productivas. Asimismo, puede aumentar el margen potencial de ganancias, al sostener la influencia del costo del mantenimiento en el final del producto, dentro del rango del 5 al $12 \%$ (Cáceres, 2009).

En este sentido, a criterio de Durán (2010), la gestión del mantenimiento se orienta a la búsqueda de metas comunes que deben ser desarrolladas y entendidas con el fin de reducir las restricciones, cuya consecución será el éxito de la empresa, y por ende del negocio. Hoy, esta meta común, se basa en la existencia de la conformidad de la calidad de los procesos y la aceptación de los resultados obtenidos, todo bajo el concepto de la excelencia en la organización.

Así, la gestión del mantenimiento se ha visto como una necesidad para toda estructura organizacional, está implícita en los procesos de producción, tanto en lo que se refiere a los productos tangibles como a los intangibles. En este sentido, es importante acotar que, donde exista infraestructura, equipos o maquinarias, la gestión de mantenimiento tendrá la fuerza de estar presente, garantizando la continuidad operacional.

Cabe destacar que las organizaciones petroleras no escapan de esta realidad, es por ello que se debe analizar la eficiencia de estas para la gestión del mantenimiento. Es importante acotar que Petróleos de Venezuela S.A. (en adelante PDVSA) posee un gran número de instalaciones, tales como: estaciones de flujo, plantas de gas y de vapor, patios de almacenamiento, almacenes, terminales, gasoductos y taladros, entre otras; cuyo funcionamiento operacional depende del buen comportamiento de diferentes equipos y maquinarias.

A objeto del presente estudio, se seleccionaron las actividades realizadas por las gerencias de perforación y rehabilitación en la región occidente, cuyo aporte dentro de las operaciones productivas de la industria petrolera es de vital importancia. Esta selección obedeció a la necesidad por conocer cómo se estaba llevando a cabo la gestión del mantenimiento en estas gerencias. Esta necesidad fue detectada mediante observación directa, como sujeto participante de sus procesos, que le ha permitido detectar desviaciones en el sistema de gestión de mantenimiento al momento de revisar sus indicadores, lo cual acarrea pérdidas tanto de dinero como tiempo. Asimismo, en entrevistas no estructuradas realizadas al personal de 
mantenimiento en las gerencias de perforación y rehabilitación de la industria petrolera en la región occidente, se pudo establecer que no se han realizado estudios que analicen la gestión de mantenimiento para conocer su situación actual. Actualmente existe: [1] Dependencia de terceros, propios y externos a PDVSA, para aplicar los distintos niveles de mantenimiento a los equipos (propios: gerencia de logística, suministro y mudanza, talleres, PPYG; externos: empresas de soldadura, pintura, certificación de equipos de izamientos, entreotras); [2] Demoras en los procesos de compras, generando inconsistencias en los stocks, provocando demoras en la ejecución del mantenimiento y labores contratadas adicionales al contrato, entre otras; [3] Inconsistencia en las órdenes de trabajo, las listas de verificación de cada mantenimiento preventivo indican realizar una actividad $X$ cuando no se cuenta con el recurso para realizarla, ejemplo de ello son los consumibles o repuestos; [4] Deficientes procesos de control para evaluar el rendimiento real de la gerencia, comparando el rendimiento real con los objetivos planeados, para tomar acciones que corrijan las diferencias entre resultados y objetivos.

Ahora bien, toda gestión posee aspectos relevantes a ser tomados en consideración al momento de querer estudiar la misma. En el caso de la gestión de mantenimiento abordada en el presente trabajo, el investigador consideró importante bajo los criterios de Mora (2009) y Garía (2009), y dado el sector de estudio, enfocarse hacia las etapas del proceso de gestión y las estrategias de mantenimiento que se cumplen en estas gerencias. Por ello, el propósito de la investigación fue analizar la gestión de mantenimiento en las gerencias de perforación y rehabilitación de la industria petrolera región occidente.

\section{METODOLOGÍA}

De acuerdo con el nivel de profundidad del trabajo que se realizó en las gerencias de perforación y rehabilitación de la industria petrolera región occidente, la investigación fue de tipo descriptiva, se enmarcó en un diseño de campo no experimental y transeccional. Para efectos de este estudio, la población quedó integrada por las gerencias de perforación y rehabilitación de la industria petrolera en la región occidente, siendo ellas 4 gerencias: Costa Oriental, Costa Occidental, Lago y Sur del Lago Trujillo. Las unidades de información fueron 26 sujetos, siendo el número total de personas a las cuales se le aplicó el instrumento, quedando representadas de la siguiente forma: 4 gerentes de división, 6 líderes y 16 supervisores de mantenimiento mayor, como se muestra en la Tabla 1.

Tabla 1. Distribución de la población.

\begin{tabular}{lcccc}
\hline \multicolumn{1}{c}{$\begin{array}{c}\text { Gerencias de perforación } \\
\text { y rehabilitación }\end{array}$} & $\begin{array}{c}\text { Gerentes de } \\
\text { división }\end{array}$ & Líderes & $\begin{array}{c}\text { Supervisores de } \\
\text { mantenimiento } \\
\text { mayor }\end{array}$ & Total \\
\hline División Costa Oriental & 1 & 0 & 3 & 4 \\
División Costa Occidental & 1 & 0 & 5 & 6 \\
División Lago & 1 & 3 & 6 & 6 \\
División Sur del Lago Trujillo & 1 & 3 & 16 & 26 \\
Total & 4 & 6 & 6 & 26 \\
\hline
\end{tabular}


En este sentido, la técnica que se utilizó para la recolección de datos fue la encuesta y el instrumento un cuestionario de 36 ítems, cuya opción de respuesta elegida debía ser marcada con un símbolo, de acuerdo con una escala específica, cerrada y dicotómica, con 2 alternativas (si o no). Dicho instrumento fue sometido a la evaluación de 2 expertos metodológicos para avalar el criterio y la construcción, y 3 expertos en el área gestión de mantenimiento, para el aval del contenido, todos con título de maestría o de doctor como característica predominante. Para calcular la confiabilidad del instrumento, se utilizó el método del Coeficiente de Küder - Richardson y se obtuvo 0,94 como valor de confiabilidad total del instrumento al incluir todos los ítems.

En este orden de ideas, para el análisis estadístico de los datos se aplicó la estadística descriptiva, que permitió caracterizar cada una de las dimensiones e indicadores, a través del uso de las frecuencias absolutas y relativas. Para la interpretación de las frecuencias relativas se consideró establecer dos baremos, tal y como se muestran en las tablas 2 y 3.

Tabla 2. Baremo de interpretación de la frecuencia.

Rango para frecuencia relativa

\begin{tabular}{cc}
\hline $\mathrm{SI} \geq 70 \%$ & Alto cumplimiento \\
$40 \% \leq \mathrm{SI}<70 \%$ & Moderado cumplimiento \\
$\mathrm{SI}<40 \%$ & Bajo cumplimiento \\
\hline
\end{tabular}

Tabla 3. Baremo de interpretación de la variable.

Rango para frecuencia relativa

$\begin{array}{cc}\mathrm{SI} \geq 70 \% & \text { Buena gestión de mantenimiento } \\ 40 \% \leq \mathrm{SI}<70 \% & \text { Regular gestión de mantenimiento } \\ \mathrm{SI}<40 \% & \text { Mala gestión de mantenimiento }\end{array}$

RESULTADOS Y DISCUSIÓN

En la Tabla 4 se presenta un resumen detallado del análisis estadístico para la dimensión etapas del proceso, cuya concentración en la respuesta afirmativa es del $78 \%$, reflejando, de acuerdo con los encuestados, alto cumplimiento de las etapas del proceso en la gestión de mantenimiento que se lleva a cabo en las gerencias de perforación y rehabilitación de la industria petrolera, región occidente. No obstante, se arriba a esta categoría en los niveles inferiores del rango en el baremo (SI $\geq 70 \%$ ). Estos resultados reflejan que, en las gerencias analizadas, según los encuestados, hay alto cumplimiento de los procesos vinculados a la planificación (91\%), asimismo con los relacionados a la organización (78\%), programación (84\%), y a la etapa de ejecución (74 \%). Sin embargo, catalogaron con moderado cumplimiento la etapa de control (61 \%) de acuerdo al baremo establecido.

Este alto cumplimiento de la dimensión etapas del proceso, valida lo expuesto por Márquez (2010), para quien el proceso de mantenimiento está compuesto por los elementos o pasos que deben 
llevarse a cabo para aplicar el mantenimiento, y estos pasos están estrechamente vinculados unos con otros. De igual manera, validan al investigador para quien las etapas del proceso de la gestión de mantenimiento son el conjunto de acciones pertinentes para su cumplimiento, que contribuyen a la efectividad de los procesos productivos, siendo estas: planificación, organización, programación, ejecución y control. En resumen, los resultados mostrados para la dimensión, ponen de manifiesto que, en las gerencias estudiadas, se otorga alto cumplimiento a las etapas del proceso, observándose oportunidades de mejora en los procesos vinculados a la organización, programación, ejecución y control.

Tabla 4. Etapas del proceso.

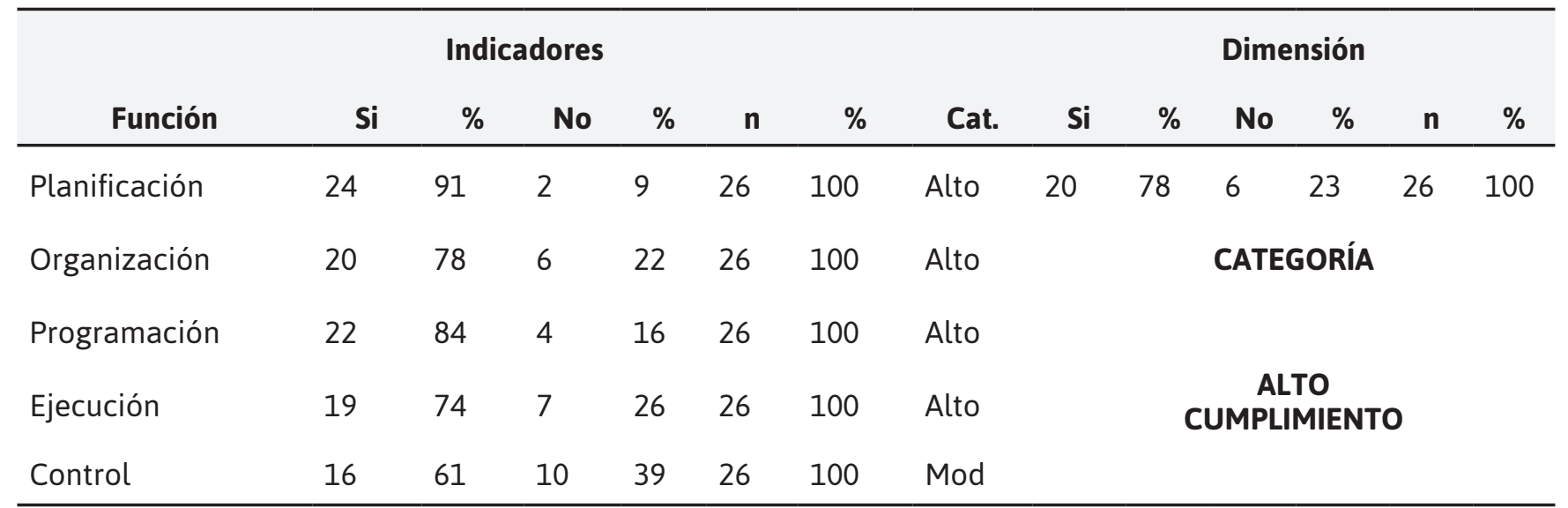

En el indicador planificación, se observa alto cumplimiento de la planificación como etapa de la gestión de mantenimiento que desarrollan las empresas mencionadas, situación develada al ubicarse en promedio el $91 \%$ de respuestas en la opción Si, ubicando el resultado en el rango $\mathrm{SI} \geq 70$ $\%$ del baremo establecido. Ahora bien, se puede apreciar que los encuestados atribuyeron alto cumplimiento a todos los procesos involucrados a la planificación; ya que, si se toma en cuenta lo que desean los clientes internos, si establecen metas en cuanto a los objetos a mantener; y se instaura un orden de prioridades para la ejecución de las acciones de mantenimiento.

Los resultados evidenciados validan los postulados de Robbins y Coulter (2005), por cuanto establecen la planificación como el proceso de definir las metas de la organización, establecer una estrategia general para alcanzarlas y trazar planes exhaustivos para integrar y coordinar el trabajo de la organización. Así mismo, alcanzan alta coincidencia con lo expuesto por el investigador cuando afirma que, la planificación del mantenimiento constituye una herramienta para mantener la continuidad de los procesos, logrando establecer las actividades requeridas, además, los recursos a utilizar y los objetivos que se pretenden alcanzar para poder generar calidad en su área operativa.

En lo concernientealindicador organización, se aprecia en promedio, que el $78 \%$ de los encuestados consideran que las actividades involucradas a los ítems se realizan con alto cumplimiento, mientras un $22 \%$ opina lo contrario. De este modo, para los encuestados, la organización del mantenimiento incluye estándares de tiempo, incluye el diseño del trabajo y, se organizan las actividades tendientes a asegurar la disponibilidad máxima planificada de los equipos a menor costo dentro de los requisitos de seguridad. 
Esto coincide con lo postulado por Duffuaa et al. (2010), para quienes la organización del mantenimiento, incluye el diseño del trabajo y estándares de tiempo. Así mismo, coincide con el investigador cuando establece que la organización en la gestión de mantenimiento es el elemento de procura y distribución de responsabilidad, fijación de tiempo, esfuerzo y costo, elementos necesarios para cada una de las actividades planificadas. De esta forma, el mantenimiento debe organizarse para dar respuesta rápida y efectiva cónsona con la realidad organizativa.

En los valores obtenidos para el indicador programación, se observa que el $84 \%$ de los encuestados respondieron de manera afirmativa a las proposiciones emitidas, indicando que las actividades relacionadas se realizan con alto cumplimiento. Mostrándose que, si se aplican programas de trabajo para ejecutar el mantenimiento; asimismo, se contemplan las prioridades de los equipos o situación requerida de mantenimiento y se utiliza un software computarizado para efectuar la programación de mantenimiento.

Esta situación valida medianamente lo expresado por Duffuaa et al. (2010), quienes aseveran que debe asegurarse que los trabajadores, piezas y materiales requeridos se encuentren disponibles antes de programar una tarea de mantenimiento. También se valida lo considerado por el investigador, al afirmar que la programación es un elemento determinante de la gestión de mantenimiento, el cual implica establecer de manera clara las secuencias en cuanto a las asignaciones de mantenimiento, así como el tiempo a ejecutarlo y los equipos que se requieren, estableciendo un orden en cada una de las solicitudes requeridas para las máquinas y herramientas.

Las respuestas correspondientes al indicador ejecución, reflejan que el $74 \%$ de los encuestados, en promedio, considera que en las gerencias bajo estudio se tiene alto cumplimiento de las actividades relacionadas al indicador. Esta situación es explicada por el comportamiento de las respuestas afirmativas apreciándose que, se asigna el recurso humano calificado para ejecutar cada mantenimiento, y se siguen normas preestablecidas al ejecutar las actividades de mantenimiento; asimismo, se ejecutan las actividades de mantenimiento según un orden de prioridad establecido.

Lo develado, en cuanto a alto cumplimiento, valida lo expuesto por Zambrano y Leal (2006), para quienes la ejecución del mantenimiento corresponde a vincular acciones administrativas con la dirección y coordinación de esfuerzos de grupos de ejecución; estos esfuerzos se generan por la planificación, organización y programación, siguiendo tanto normas como procedimientos preestablecidos, para el logro de las metas dentro de los objetivos fijados para el mantenimiento. También son congruentes con la posición del investigador, quien asume la ejecución como un elemento fundamental para que las actividades puedan realizarse de acuerdo a lo establecido; por ello, corresponde vincular cada una de las acciones de la organización con los esfuerzos de un personal responsable, generando un mejor compromiso al momento de ejecutar el mantenimiento.

En lo referido al indicador control, el cual obtuvo la categoría de moderado cumplimiento, se aprecia que el $61 \%$ de los encuestados consideraron que, en las gerencias bajo estudio, se da moderado cumplimiento a las actividades relacionadas. En este sentido, comparan el porcentaje de mantenimiento realizado con el programado y analizan la información registrada sobre los mantenimientos realizados para detectar acciones correctivas.

Lo mostrado valida medianamente a Martínez (2007), quien establece que el control será el resultado de evaluar un número de indicadores

ISSN: 2788 - 6557| ISNN-L: 2788 - 6557
Volumen 3 | No. 2 | Julio - diciembre 2021
www.revistapanel.org


capaces de reflejar cantidad, costo, eficiencia y disponibilidad en los trabajos ejecutados. Al igual que es medianamente coincidente con lo expresado por el investigador, cuando afirma que el control se enfoca en evaluar y corregir el desempeño de las actividades de mantenimiento de los subordinados para asegurar que los objetivos y planes de la organización se están llevando a cabo.

La Tabla 5 muestra el resumen para la dimensión estrategias de mantenimiento, reflejando alto cumplimiento de estas actividades en la gestión de mantenimiento que se llevan a cabo en las gerencias estudiadas, indicado por una concentración del 74 \% en la opción afirmativa. Al analizar los porcentajes de respuestas afirmativas alcanzados para la dimensión en cada uno de sus indicadores, se percibe que, las estrategias de mantenimiento correctivo y preventivo poseen alto cumplimiento en la gestión de mantenimiento que desarrollan, con concentraciones de respuestas afirmativas que así lo demuestran, correctivo (78 \%) y preventivo (88\%). Mientras, las estrategias de mantenimiento predictivo y proactivo ostentaron moderado cumplimiento (63 $\%$ y $67 \%$; respectivamente).

Al detalle se aprecia que todas las estrategias de mantenimiento, consideradas en esta investigación: correctivo, preventivo, predictivo, y proactivo, se ubicaron en la categoría de alto cumplimiento. No obstante, tanto en el correctivo, como el predictivo y en el proactivo se observan actividades ubicadas en la categoría bajo y moderado cumplimiento, lo cual es indicativo de oportunidades de mejora en estos aspectos. A manera de resumen, se establecieron las estrategias de mantenimiento utilizadas con mayor frecuencia hoy en día, observándose que en las gerencias de perforación y rehabilitación de la industria petrolera región occidente, se posee alto cumplimiento de estas estrategias, acotándose que la elección del tipo de mantenimiento dependerá en gran medida de las estrategias planteadas en la planificación del mantenimiento.

Los resultados validan lo expuesto por Duffuaa et al. (2010), pues las estrategias de mantenimiento son aplicadas para optimizar la producción y disponibilidad de la planta sin que se comprometa la seguridad y el ambiente. La combinación en forma correcta de las estrategias influye en el desarrollo de una gestión de mantenimiento eficiente y eficaz. En síntesis, también validan al investigador cuando considera que, existen diversas definiciones sobre los tipos de estrategias de mantenimiento aplicable en situaciones operacionales, lo que dificulta la unificación de criterios al respecto. No obstante, independientemente de la percepción de cada autor, el tipo de mantenimiento se puede establecer en función de estrategias de mantenimiento que relacionan el momento de ocurrencia de fallas y el momento de ejecución de la labor de mantenimiento.

En relación al indicador mantenimiento correctivo, se evidencia que el $78 \%$ de los encuestados consideraron la opción afirmativa, indicando alto cumplimiento del mantenimiento correctivo en la gestión de mantenimiento que llevan a cabo las gerencias estudiadas. Estos resultados están delineados por el nivel de cumplimiento asignado a cada una de las actividades medidas por medio de los reactivos vinculados a la medición del indicador. Por consiguiente, se aprecia que los encuestados realizan chequeos rutinarios para corregir fallas aplicando mantenimiento correctivo, corrigen las fallas que se presentan en el momento específico y ejecutan mantenimiento correctivo al momento de presentarse la falla. No obstante, estas gerencias no poseen un plan de contingencia ante un evento que amerite ejecutar un mantenimiento correctivo.

Los resultados mostrados validan lo postulado por García (2009), para quien en la estrategia 
centrada en el mantenimiento correctivo se deja que el equipo o sistema continúe operando hasta que el mismo falle por alguno de sus componentes, que lo inhabilite, para luego tomar la acción de ser reemplazado. Asimismo, validan al investigador quien opina que el mantenimiento correctivo no se puede eliminar en su totalidad, por lo tanto, una gestión correcta extraerá conclusiones de cada parada e intentará realizar la reparación de manera definitiva, ya sea en el mismo momento o programando un paro, para que esa falla no se repita. En consecuencia, es importante tener en cuenta con respecto al análisis de la política de mantenimiento a implementar, que en algunas máquinas 0 instalaciones, el mantenimiento correctivo sería el sistema más rentable.

Tabla 5. Estrategias de mantenimiento.

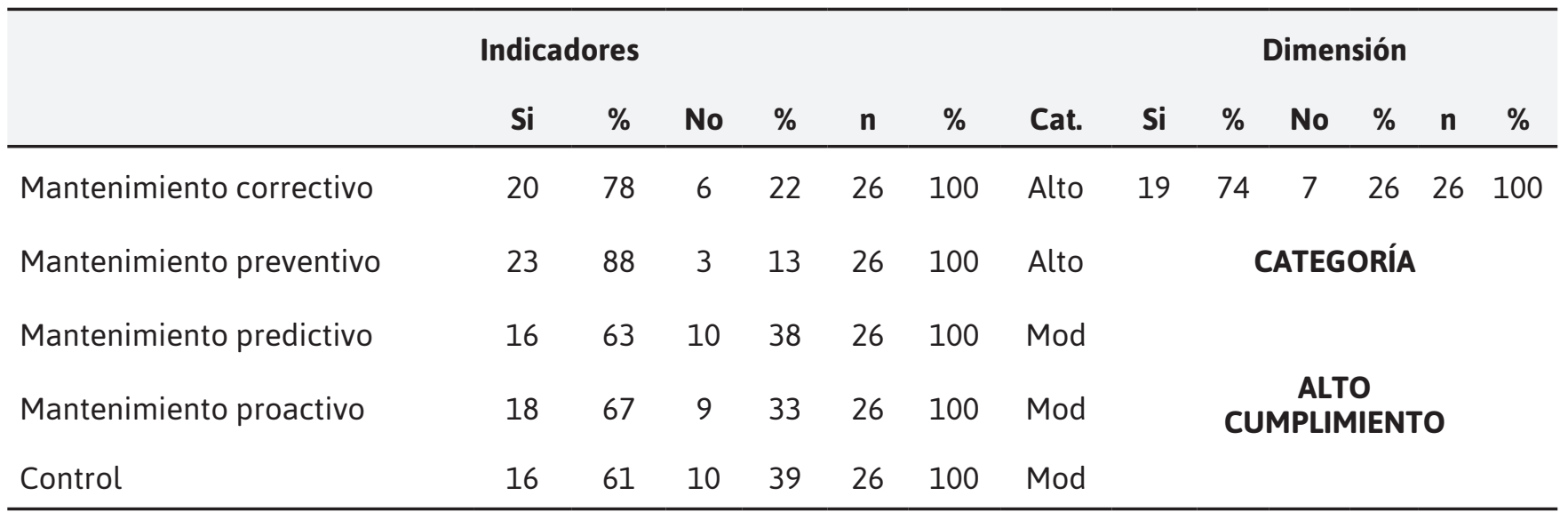

En cuanto al indicador mantenimiento preventivo se observa que alcanzó un porcentaje de respuestas en la opción afirmativa del $88 \%$, indicando que en las gerencias analizadas se tiene alto cumplimiento del mantenimiento preventivo en su gestión de mantenimiento. Esta situación es explicada por el alto cumplimiento otorgado a todas las actividades vinculadas al indicador. De este modo, para los encuestados se aplica el mantenimiento siguiendo un programa de frecuencia; asimismo se analizan las condiciones de los equipos mientras se encuentran funcionando y se cuentan con un historial del mantenimiento realizado a todos los equipos.

Los resultados logran alta congruencia con Nava (2008), quien concibe el mantenimiento preventivo como la actividad planeada y programada para ajustar, reparar o cambiar partes en equipos antes de que ocurra la falla o daños mayores, reduciendo así los costos generados. A juicio del investigador, dado que se tiene alto cumplimiento de este tipo de mantenimiento, en estas gerencias se están aprovechando las oportunidades que ofrece el mismo, lo cual involucra utilizar todos los medios disponibles, incluso los estadísticos, para determinar la frecuencia de las inspecciones, revisiones, sustitución de piezas claves, probabilidad de aparición de averías, vida útil u otras. En síntesis, consiste en programar revisiones de equipos, apoyándose en la experiencia e históricos obtenidos de las máquinas y equipos.

Con relación al indicador mantenimiento predictivo correspondiente a la dimensión estrategias de mantenimiento, se evidencia que a criterio del $63 \%$ de los encuestados, la estrategia predictiva se da con moderado cumplimiento. Desde el punto de vista de los resultados, si se considera importante contar con una visión de la condición de los equipos, detectan el mal estado de un componente previo al inicio de su operación, y no detectan las fallas sin necesidad de detener el funcionamiento de los equipos. 
Los resultados muestran que, las actividades del mantenimiento predictivo poseen moderado cumplimiento dentro de la gestión de mantenimiento, por ello, se deduce que hay mediana congruencia con lo expuesto por Nava (2009), para quien esta estrategia engloba todas las actividades que permiten detectar fallas mecánicas $\mathrm{u}$ operacionales de los equipos en su fase inicial, mediante análisis realizados con equipos especiales. Las fallas son detectadas sin necesidad de detener el funcionamiento de los equipos. De igual manera, alcanzan mediana coincidencia con lo establecido por el investigador, cuando afirma que este tipo de mantenimiento se basa en actividades de control de la condición de funcionamiento de los equipos, de manera continua o a intervalos, y con base en los resultados, se planifican y realizan acciones de reparación o cambios.

En referencia al indicador mantenimiento proactivo, según la opinión de los encuestados indican que en las gerencias estudiadas se tiene moderado cumplimiento de esta estrategia en su gestión de mantenimiento, arribando este indicador a una frecuencia de $67 \%$ de respuestas afirmativas. De tal forma, las gerencias elaboran análisis de las causas fundamentales de las fallas; y se realiza monitoreo del cambio en la tendencia de los parámetros considerados como causa de falla. Sin embargo, no aplican técnicas proactivas para alargar la disponibilidad de las máquinas.

Estos resultados evidencian mediana congruencia con García (2009), quien señala al tipo proactivo como una gran cantidad de técnicas para alargar la disponibilidad de las máquinas. La idea principal de un mantenimiento proactivo es el análisis de las causas raíz de las fallas, así estas causas se pueden remediar y los mecanismos de falla que se puedan eliminar gradualmente, mejorarán la operación de las máquinas. Asimismo, se valida moderadamente al investigador cuando afirma que, esta estrategia es utilizada para mejorar el rendimiento de cualquier organización, considerando el estudio de probabilidad de falla y disminuyendo el riesgo de aparición de la misma. Todo esto debe cumplirse para tratar de obtener costos totales mínimos de operación, operando en buenas condiciones durante un porcentaje de tiempo óptimo.

Para concluir el análisis de los resultados se presentanacontinuaciónloshallazgosencontrados para la variable en estudio. Con base a las tablas precedentes (resultados de las dimensiones), se elaboró la Tabla 6 con el propósito de evidenciar los resultados arrojados para la variable gestión de mantenimiento. Como puede apreciarse, los resultados obtenidos demostraron que los gerentes, líderes y supervisores de las gerencias de perforación y rehabilitación de la industria petrolera, catalogan la gestión de mantenimiento con la categoría de buena, situación que se refleja al obtenerse una concentración de respuestas en la opción afirmativa del $76 \%$.

Tabla 6. Gestión de mantenimiento.

\begin{tabular}{lccccccccccccc}
\hline & \multicolumn{3}{c}{ Dimensiones } & & 1 & & & \multicolumn{4}{c}{ Variable } \\
& Si & $\%$ & No & $\%$ & $\mathbf{n}$ & $\%$ & Cat. & Si & $\%$ & No & $\%$ & $\mathbf{n}$ & $\%$ \\
\hline Etapas del proceso & 20 & 78 & 6 & 23 & 26 & 100 & Alto & 19 & 76 & 7 & 24 & 26 & 100 \\
Estrategias de mantenimiento & 19 & 74 & 7 & 26 & 26 & 100 & Alto & & $\begin{array}{c}\text { CATEGORÍA } \\
\text { BUENA GESTIÓN DE } \\
\text { MANTENIMIENTO }\end{array}$ \\
\hline
\end{tabular}


Cabe resaltar que la variable gestión de mantenimiento fue medida a través de 2 dimensiones, como se refleja en la Tabla 3. Estas se midieron cuantitativamente, con un cuestionario basado en una escala dicotómica, y se evidenció un promedio del $76 \%$ en la opción afirmativa para la variable, ubicándola, de acuerdo al baremo diseñado para la variable, en buena gestión de mantenimiento, situación que es producto del alto cumplimiento de las etapas del proceso (78 \%) y las estrategias de mantenimiento (74\%).

Estos resultados concuerdan en gran medida con lo planteado por Rodríguez (2008), para quien la gestión de mantenimiento es el conjunto de actividades de diseño, planificación y control que tienen por objeto minimizar los costos asociados al mal funcionamiento de los equipos; además de las actividades típicas de mantenimiento, debe incluirse la formación del personal.

Así mismo, los resultados validan lo expuesto por Zambrano y Leal (2007), cuando aseguran que, la gestión de mantenimiento es un proceso sistémico donde a través de una serie de medidas organizativas se pueden planear las acciones de las actividades por medio de procedimientos que lleven un orden o secuencia lógica de esta función, a fin de conseguir un constante y adecuado desempeño de los equipos pertenecientes al sistema productivo, esto con la finalidad de identificar los pasos a seguir y prever las posibles desviaciones que se puedan presentar en el desarrollo de estas actividades.

Sin embargo, a juicio del investigador, hay que resaltar que la gestión analizada alcanzó la categoría de buena en los niveles más bajos de la categoría en el baremo (SI $\geq 70 \%$ ) mostrando oportunidades de mejora en todas sus dimensiones. No obstante, estas consideraciones permiten deducir que la gestión analizada debe ser revisada a fin de ser mejorada continuamente, con miras a fortalecerse y mantenerse en el tiempo.

\section{CONCLUSIONES}

En lo concerniente a las etapas del proceso de gestión de mantenimiento que se cumplen en las gerencias de perforación y rehabilitación de la industria petrolera en la región occidente, se concluye que estas gerencias poseen alto cumplimiento de estas etapas en su gestión de mantenimiento, situación evidenciada en alto cumplimiento de los procesos vinculados a la planificación, organización, programación y a la etapa de ejecución. Sin embargo, catalogaron con moderado cumplimiento la etapa de control.

Debe mencionarse que aun con esta categoría de alto cumplimiento alcanzada en las etapas del proceso, se detectaron actividades que se realizan con moderado cumplimiento, a juicio de los sujetos encuestados, específicamente en todas las etapas, a excepción de la planificación donde todas las actividades fueron catalogadas con alto cumplimiento. Estos aspectos deben ser revisados por la gerencia, en virtud de la importancia que tienen las etapas del proceso en la gestión de mantenimiento, debido a su efecto en toda la organización; dado que de ello dependerá el logro de los objetivos.

En cuanto a las estrategias de mantenimiento que se cumplen en las gerencias de perforación y rehabilitación de la industria petrolera región occidente, se concluye que estas estrategias alcanzanalto cumplimiento enlagestiónanalizada. Destacándose los mantenimientos correctivo y preventivo que ostentaron alto cumplimiento, mientras que el resto de las estrategias (predictivo y proactivo) se posicionaron en la categoría de moderado cumplimiento.

Estos aspectos, a juicio de los encuestados, muestran oportunidades de mejora, en específico las estrategias de mantenimiento correctivo, predictivo y proactivo, evidenciaron actividades con moderado y bajo cumplimiento, motivo por el cual la gerencia debe revisarlos, dada la importancia que tienen las estrategias en la gestión de mantenimiento. 
Atendiendo a las consideraciones precedentes, con respecto a la gestión de mantenimiento que se desarrolla en las gerencias de perforación y rehabilitación de la industria petrolera, región occidente, se concluye que estas gerencias, según la percepción de los encuestados, ostentan buena gestión de mantenimiento. Situación que es producto del alto cumplimiento de las etapas del proceso y las estrategias de mantenimiento. No obstante, esta gestión de mantenimiento se cataloga como buena en los niveles más bajos de esta categoría. En función de lo cual, se les presenta la necesidad de revisar sus acciones, donde es imperante la implementación de mecanismos para gestionar el mantenimiento, basados en la revisión de los aspectos señalados en estas conclusiones.

Las situaciones descritas conllevan a concluir que se ratifica la posición inicial del investigador, cuando se propone analizar la gestión de mantenimiento que se desarrolla en las gerencias de perforación y rehabilitación de la industria petrolera, a fin de comprobar si en estas gerencias se presentan debilidades relacionadas con demoras en los procesos de compras, inconsistencia en las órdenes de trabajo y falta de estrategias de control para evaluar el rendimiento real de la gestión.

\section{REFERENCIAS}

Cáceres, M. (2009). Cómo incrementar la competitividad del negocio mediante estrategias para gerenciar el mantenimiento. (EN LÍNEA). Disponible en http://www. mantenimientomundial.com/sites/mm/notas/ competitividad.pdf

Duffuaa, S., Raouf, A. y Campbell, J. (2010). Sistemas de Mantenimiento. Planificación y Control. Editorial Limusa. México

Durán, J. (2010). Gestión de mantenimiento bajo estándares internacionales como PAS 55 ASSET Management. The Woodhouse Parthership. Consultado agosto 2015. Disponible en: http:// www.mantenimientomundial.com/sites/mm/ notas/PAS55.pdf/
García, S. (2009). Organización y gestión integral de mantenimiento. Tercera Edición. Ediciones Díaz de Santos S.A. Madrid. España

Márquez, M. (2010). Manual de la Ingeniería de Calidad. Gestión de mantenimiento. Editorial Panapo. Caracas. Venezuela

Martínez, L. (2007). Organización y planificación de sistemas de mantenimiento. Centro de altos estudios gerenciales. Instituto Superior de Investigación y Desarrollo. Segunda Edición. Caracas. Venezuela.

Mora. H. (2011). Mantenimiento. Planeación, ejecución y control. Editorial Alfa-Omega Grupo Editor, S.A. México D.F. México

Nava, J. (2008). Teoría del mantenimiento. Segunda Edición. Universidad de los Andes. Consejo de publicaciones. Mérida. Venezuela

Nava, J. (2009). Aplicación práctica de la teoría de mantenimiento. Segunda edición. Editorial Consejo de publicaciones de la Universidad de los Andes. Venezuela

Robbins, S. y Coulter, M. (2005). Administración. Octava edición. Editorial Pearson Prentice Hall. México

Rodríguez, J. (2008). Gestión de mantenimiento. Introducción a la teoría del mantenimiento. Casos empresariales. Documento en línea. Disponible en http://www.scribd.com/ doc/7497765/gestion-del-mantenimiento

Zambrano, S. y Leal, S. (2006). Manual práctico de gestión de mantenimiento. Fondo Editorial UNET. San Cristóbal. Venezuela

Zambrano, S. y Leal, S. (2007). Fundamentos Básicos de Mantenimiento. Segunda edición. Fondo Editorial UNET. Táchira. Venezuela 